\title{
Oxygen-derived Free Radicals Stimulate Osteoclastic Bone Resorption in Rodent Bone In Vitro and In Vivo
}

I. Ross Garrett, Brendan F. Boyce, Richard O. C. Oreffo, Lynda Bonewald, James Poser, and Gregory R. Mundy

Department of Medicine/Endocrinology and Metabolism, University of Texas Health Science Center, San Antonio, Texas $78284-7877$

\section{Abstract}

The mechanisms by which bone resorbing osteoclasts form and are activated by hormones are poorly understood. We show here that the generation of oxygen-derived free radicals in cultured bone is associated with the formation of new osteoclasts and enhanced bone resorption, identical to the effects seen when bones are treated with hormones such as parathyroid hormone (PTH) and interleukin 1 (IL-1). When free oxygen radicals were generated adjacent to bone surfaces in vivo, osteoclasts were also formed. PTH and IL-1-stimulated bone resorption was inhibited by both natural and recombinant superoxide dismutase, an enzyme that depletes tissues of superoxide anions. We used the marker nitroblue tetrazolium (NBT) to identify the cells that were responsible for free radical production in resorbing bones. NBT staining was detected only in osteoclasts in cultures of resorbing bones. NBT staining in osteoclasts was decreased in bones coincubated with calcitonin, an inhibitor of bone resorption. We also found that isolated avian osteoclasts stained positively for NBT. NBT staining in isolated osteoclasts was increased when the cells were incubated with bone particles, to which they attach. We confirmed the formation of superoxide anion in isolated avian osteoclasts using ferricytochrome $c$ reduction as a method of detection. The reduction of ferricytochrome $c$ in isolated osteoclasts was inhibited by superoxide dismutase.

Our results suggest that oxygen-derived free radicals, and particularly the superoxide anion, are intermediaries in the formation and activation of osteoclasts. ( J. Clin. Invest. 1990. 85:632-639.) bone resorption • oxygen radicals • osteoclasts

\section{Introduction}

Osteoclastic bone resorption is a complex process that involves the release of mineral from bone and then degradation of the proteinaceous bone matrix (for review, see 1). The signaling mechanisms responsible for formation and activation of the osteoclast are poorly understood. Since it is likely that osteoclasts are derived from cells of the monocyte-macrophage lineage (1), and cells in this family generate oxygen-derived free radicals that have been linked to multiple cellular effects as well as connective tissue degradation $(2,3)$, we questioned

Address reprint requests to Dr. Garrett, Department of Medicine, Division of Endocrinology and Metabolism, University of Texas Health Science Center, 7703 Floyd Curl Drive, San Antonio, TX 78284-7877.

Received for publication 29 December 1987 and in revised form 24 July 1989.

J. Clin. Invest.

(c) The American Society for Clinical Investigation, Inc.

0021-9738/90/03/0632/08 \$2.00

Volume 85, March 1990, 632-639 whether formation and activation of bone resorbing osteoclasts was associated with oxygen-derived free radical generation.

\section{Methods}

Fetal long bone assay. Pregnant rats at the 18 th $\mathrm{d}$ of gestation were injected with $200 \mu \mathrm{Ci}$ of ${ }^{45} \mathrm{Ca}$. The mothers were killed on the 19 th $\mathrm{d}$ of gestation, and the fetuses were removed. The mineralized shafts of the radii and ulnae were dissected free of surrounding tissue and cartilage and incubated in BGJb medium (Gibco Laboratories, Grand Island, $\mathrm{NY}$ ) for $24 \mathrm{~h}$ at $37^{\circ} \mathrm{C}$ in a humidified atmosphere of $5 \% \mathrm{CO}_{2}$ and $95 \%$ air to allow for the exchange of loosely complexed ${ }^{45} \mathrm{Ca}$. Bones were then cultured for $48-120 \mathrm{~h}$ in BGJb medium supplemented with 1 $\mathrm{mg} / \mathrm{ml}$ bovine serum albumin (RIA grade; Sigma Chemical Co., St. Louis, MO) containing control or test substances. Bone resorbing activity was measured as the percentage of the total ${ }^{45} \mathrm{Ca}$ released into the medium and was expressed as a treated-to-control ratio. Statistical significance was determined with Student's $t$ test for unpaired data. This technique has been described in detail previously (4).

Neonatal mouse calvarial assay. This assay has also been described before (5). Pregnant ICR Swiss white mice (17th d gestation) were injected with $25 \mu \mathrm{Ci}$ of ${ }^{45} \mathrm{Ca} .4 \mathrm{~d}$ after birth the front and parietal bones were removed from the neonatal mice by microdissection and split along the sagittal suture. After a $24-\mathrm{h}$ preincubation period in BGJb medium (Irvine Scientific, Santa Ana, CA) at $37^{\circ} \mathrm{C}$ in a humidified atmosphere of $5 \% \mathrm{CO}_{2}$ and $95 \%$ air to allow for the exchange of loosely complexed ${ }^{45} \mathrm{Ca}$, the half-calvariae were transferred into fresh medium containing test or control substances for a further 72-96 h. Four halfcalvariae were used in each experimental group and the bone resorbing activity was expressed as treated-to-control ratios as described above. In these experiments, statistical differences were evaluated using Student's $t$ test for unpaired data.

Isolation and culture of chick osteoclasts. Avian osteoclasts were isolated from medullary bone of egg-laying white leghorn hens (King's Wheel, Mt. Vernon, OH). Hens were fed a hypocalcemic diet (Purina Test Diet Co., Shelbyville, IN) for 2 wk as previously described (6-8). In brief, bone marrow osteoclast suspensions from the medullary bone were filtered through Nytex cloth $(110 \mu \mathrm{m})$ (Tetko, Elmsford, NY), centrifuged for $5 \mathrm{~min}$ at $1,200 \mathrm{rpm}$ and the cell pellet suspended in $0.2 \% \mathrm{NaCl}$ and incubated on ice for $2 \mathrm{~min}$ to remove red blood cells. The cells were then layered onto $70 \%$ heat-inactivated fetal bovine serum (FBS) (Gibco Laboratories) and allowed to stand on ice for 1-2 $\mathrm{h}$, after which the sedimented cells were washed and resuspended in $\alpha$-MEM (Gibco Laboratories) containing $10 \%$ FBS, $100 \mathrm{U} / \mathrm{ml}$ penicillin, $100 \mu \mathrm{g} / \mathrm{ml}$ streptomycin, and $3 \mu \mathrm{g} / \mathrm{ml}$ of arabinose- $\beta$ - $d$-cytosine furanoside to inhibit mitosis of nonosteoclastic cells. The osteoclasts were then counted and plated into 24-well (Costar, Cambridge, MA) culture dishes. These cell cultures were incubated at $37^{\circ} \mathrm{C}$ in a $5 \% \mathrm{CO}_{2}$ humidified atmosphere for $48 \mathrm{~h}$, after which the nonadherent cells were removed by washing. The adherent osteoclasts were then incubated for a further $48 \mathrm{~h}$ in fresh media with or without bone particles (50-100 $\mu \mathrm{m})$.

Generation of oxygen-derived free radicals in bone organ cultures. Several manipulations of both the fetal rat long bone culture system and the neonatal mouse calvarial culture system were used to determine whether the generation of free radicals was involved in osteoclastic bone resorption. Firstly, xanthine oxidase and its substrate 
xanthine were used to generate oxygen-derived free radicals (9) in bone organ cultures. To confirm that the bone resorption associated with generation of oxygen-derived free radicals by xanthine oxidase/xanthine was in fact mediated by the free radicals, the enzymes superoxide dismutase and catalase were used. The reduction of oxygen by the monovalent pathway produces the superoxide anion $\left(\mathrm{O}_{2}^{-}\right)$and hydrogen peroxide $\left(\mathrm{H}_{2} \mathrm{O}_{2}\right)$, and if these are not scavenged, hydroxyl radical $\left(\mathrm{OH}^{\circ}\right)(3,9,10)$. Superoxide dismutase is a ubiquitous enzyme, which depletes tissues of superoxide anions by converting the superoxide anions to hydrogen peroxide. Catalase consumes hydroxyl radicals by metabolizing hydrogen peroxide to oxygen. These enzymes can be utilized to confirm the requirement of these radicals in the resorption process, and to distinguish the importance of superoxide anions relative to hydroxyl ions.

Because calcitonin is a hormone that specifically inhibits osteoclast activity, it was used to determine whether the effects of generation of oxygen-derived free radicals on bone resorption in these organ cultures was dependent on resorbing osteoclasts.

In vivo generation of oxygen-free radicals at bone surfaces. $10 \mu \mathrm{l}$ of a $25-\mathrm{mM}$ solution of xanthine and $10 \mu \mathrm{l}$ of a $5-\mathrm{U} / \mathrm{ml}$ solution of xanthine oxidase was injected locally into the subcutaneous tissue over the right side of the calvariae of 5-wk-old mice three times a day for $3 \mathrm{~d}$. The mice were then killed, the calvariae removed and fixed in phosphate-buffered formalin, and then processed for histology.

Quantification of morphologic changes in bone. Sections of calvarial bones from both in vitro organ cultures and from mice treated with xanthine oxidase/xanthine in vivo were examined histologically. The bone matrix area between the lambdoid and coronal sutures was measured and the number of osteoclasts per square millimeter of bone was counted using the Bioquant Image Analysis System (R \& M Biometrics, Inc., Nashville, TN). The extent of resorption along the inner and outer bone surfaces was also measured. This histologic technique has been described in detail previously $(11,12)$.

Nitroblue tetrazolium $(N B T)^{l}$ staining of calvaria and isolated osteoclasts. NBT (Sigma grade III) $20 \mathrm{mg}$ was dissolved $10 \mathrm{ml}$ phosphate-buffered saline at pH 7.4 (11). Calvariae after $72 \mathrm{~h}$ of culture were incubated in this NBT solution at $37^{\circ} \mathrm{C}$ in a $5 \% \mathrm{CO}_{2}$ humidified atmosphere for $30 \mathrm{~min}$ after which they were fixed in $10 \%$ phosphatebuffered formalin. Macroscopic examination of the cultured calvariae was performed using a Nikon Diaphot phase contrast inverted microscope at low power $(\times 5)$. The calvariae were examined for resorption areas (which could be seen as discrete areas in the thin bones) and the numbers of NBT-positive cells $/ \mathrm{mm}^{2}$ were counted. The calvariae were then processed for histology (decalcified in 14\% EDTA, dehydrated in graded alcohols and embedded in paraffin). Four 5- $\mu \mathrm{M}$ thick, nonconsecutive step-sections were cut and stained using hematoxylin and eosin. The total number of osteoclasts and NBT-positive cells were counted/section at four different levels through the calvariae.

The isolated avian osteoclasts that were cultured with or without bone particles were also fixed and examined directly after incubation with NBT.

Chemicals and reagents. Xanthine oxidase (grade 1), xanthine, superoxide dismutase (type 1 , from bovine erythrocytes), recombinant human superoxide dismutase (Chiron Corp., Emeryville, CA; lot C9D01CR), NBT (grade III), cytochrome $c$ and catalase were purchased from Sigma Chemical Co. Human PTH (1-34) was purchased from Bachem Corp. (Torrance, CA). Superoxide dismutase and catalase were prepared in BGJb medium at 100-fold concentration and diluted as needed. Salmon calcitonin (synthetic) was provided by Rorer, Inc. (Philadelphia, PA). All solutions were made fresh immediately before use.

\section{Results}

We found that concentrations of $0.05 \mathrm{mU} / \mathrm{ml}$ xanthine oxidase with xanthine at $25 \mu \mathrm{M}$ caused resorption in both fetal rat

1. Abbreviation used in this paper: NBT, nitroblue tetrazolium. long bone and neonatal mouse calvariae organ cultures (Fig. 1). The generation of superoxide radicals in this system was confirmed using a cytochrome $c$ reduction assay (10) in phosphate-buffered saline. Using xanthine at a concentration of 25 $\mu \mathrm{M}$, we found that $50 \mathrm{mU} / \mathrm{ml}$ xanthine oxidase produced 87 $\mathrm{nM} / \mathrm{ml}$ per $\mathrm{h}$ of $\mathrm{O}_{2}^{-}$and $5 \mathrm{mU} / \mathrm{ml}$ xanthine oxidase produced 8 $\mathrm{nM} / \mathrm{ml}$ per $\mathrm{h}$ of $\mathrm{O}_{2}^{-}$under these experimental conditions. We found no effect of heat-inactivated xanthine oxidase or its substrate xanthine on bone resorption. Similarly, there was no effect of uric acid, the product of the enzyme reaction, on bone resorption (data not shown).

Bone resorption caused by the generation of oxygen-derived free radicals using the xanthine oxidase/xanthine system was inhibited by superoxide dismutase $(6 \mu \mathrm{M})$ but not affected by catalase $(6 \mu \mathrm{M})$ (Fig. 2). These results suggest that the superoxide anion rather than the hydroxyl anion is the major reactive oxygen intermediate responsible for the bone resorption that occurs in this system. Calcitonin also inhibited bone resorption in mouse calvariae, which was stimulated by generation of oxygen-derived free radicals, suggesting the involvement of functioning osteoclasts in radical-related bone resorption (Fig. 2). However, indomethacin (1 $\mu \mathrm{M})$, an inhibitor of prostaglandin synthesis, had no significant effect on radicalmediated bone resorption (data not shown), suggesting that prostaglandins are not directly involved in this process.

Treatment of calvarial bone organ cultures with xanthine/ xanthine oxidase caused a dramatic increase in the number of osteoclasts and the extent of resorption surfaces in histologic sections (Table I, Fig. 3), and these changes were accompanied by a significant reduction in the amount of bone matrix (Table I, Fig. 1). These changes were similar to those observed when calvariae were treated with PTH or IL-1. When superoxide dismutase was added to bones treated with xanthine/xanthine oxidase, the stimulatory effects of xanthine/xanthine oxidase on osteoclast numbers and resorption surfaces was significantly inhibited (Table I).

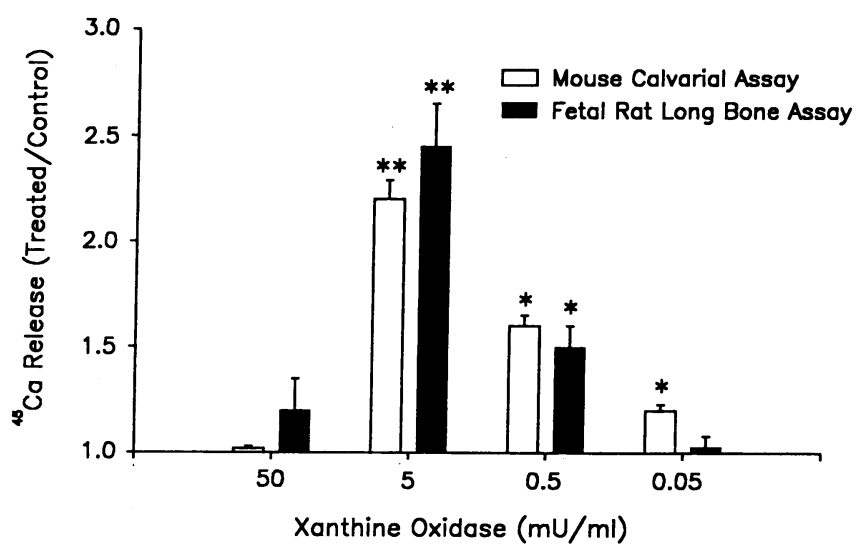

Figure 1. The effects of oxygen-derived free radical generation by xanthine oxidase/xanthine on organ cultures of bone. The bone cultures were neonatal mouse calvariae and fetal rat long bones. Neonatal mouse calvariae were incubated for $72 \mathrm{~h}$, and fetal rat long bones were incubated for $120 \mathrm{~h}$. Bone resorbing activity is expressed as treated-to-control ratios of ${ }^{45} \mathrm{Ca}$ release. Bones incubated in control media released $16.7 \pm 0.7$ (calvariae) and $18.3 \pm 0.8$ (long bones) of previously incorporated ${ }^{45} \mathrm{Ca}$. ${ }^{* *}$ Significantly $>1.0, P<0.001$. ${ }^{*}$ Significantly $>1.0, P<0.01$. 


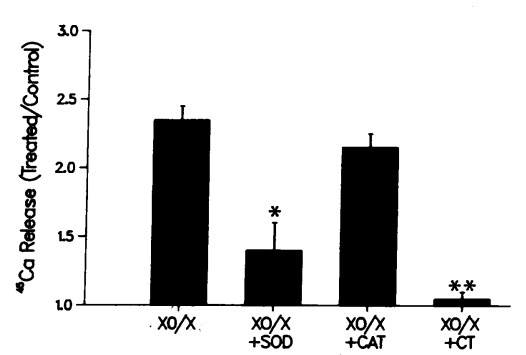

Similar responses were seen in vivo in response to radical generation. We found an increase in osteoclast numbers and in the extent of the resorption surface along the interface between bone and bone marrow in calvariae from mice treated with xanthine/xanthine oxidase (Table I, experiment 1). These findings are similar to those observed when we injected IL- $1 \alpha$ ( $2.5 \mu \mathrm{g}$ once a day for $3 \mathrm{~d}$ ) over the calvariae of young mice (unpublished observations).
Figure 2. Effects of superoxide dismutase $(6$ $\mu \mathrm{M})$, catalase $(6 \mu \mathrm{M})$, and salmon calcitonin $(10 \mathrm{U} / \mathrm{ml})$ on bone resorption stimulated by oxygen-derived free radicals generated by xanthine oxidase/xanthine $(5 \mathrm{mU} / \mathrm{ml}) .{ }^{* *} \mathrm{Sig}$ nificantly $<\mathrm{XO} / \mathrm{X}$ alone, $P<0.001$. *Significantly $<\mathrm{XO} / \mathrm{X}$ alone, $P<0.05$.
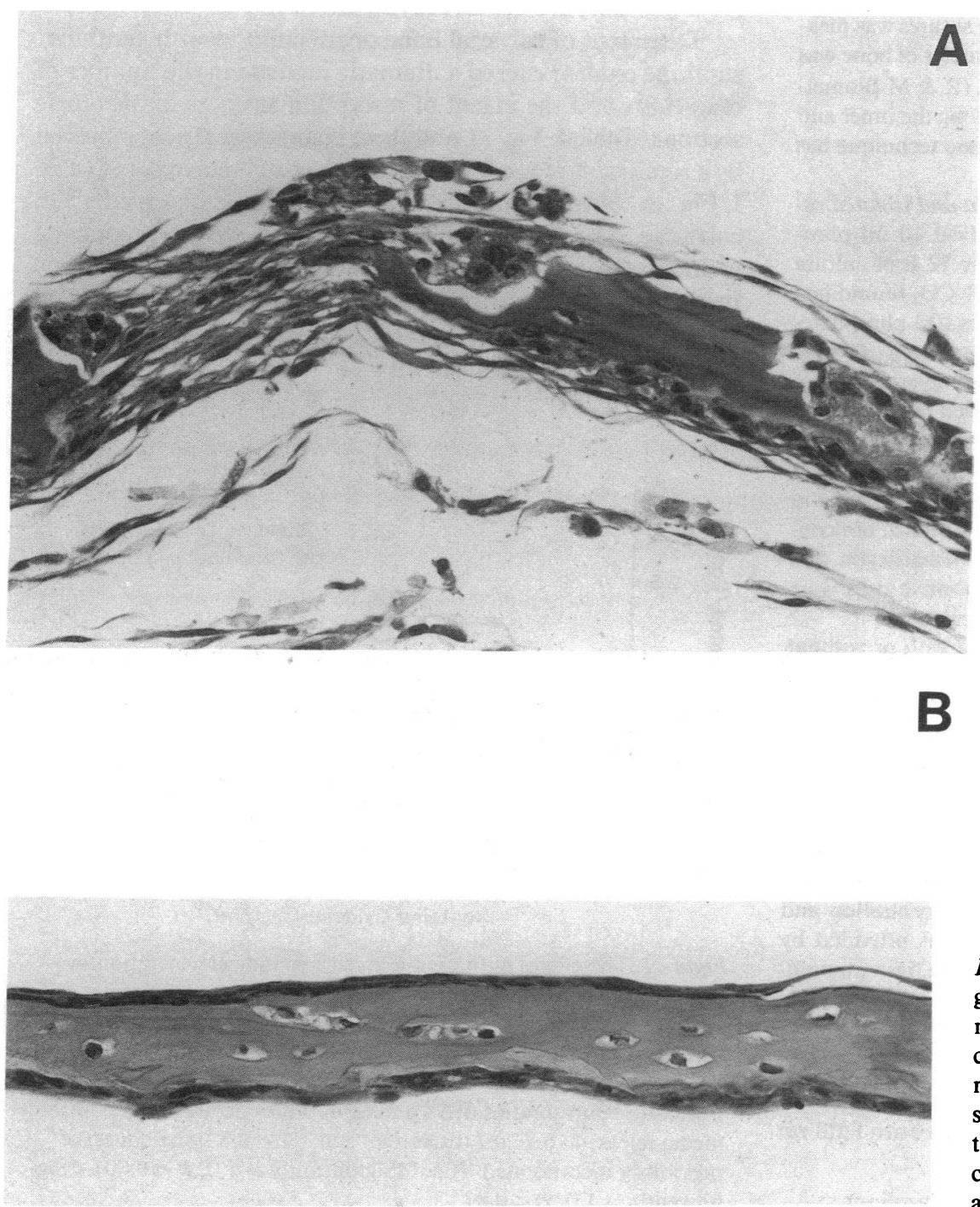

Figure 3. Effects of oxygen-derived free radical generation by xanthine oxidase/xanthine (50 $\mathrm{mU} / 0.25 \mu \mathrm{mol}$ ) on cultures of neonatal mouse calvariae $(A)$ and bones incubated with control media $(B)$. Calcified bone matrix is being resorbed by multinucleated osteoclasts in a bone treated with xanthine oxidase/xanthine. (Decalcified sections stained with hematoxylin and eosin, $\times 312$.) 
Table I. Effects of Radical Generation on Morphological Indices of Bone Resorption in Organ Cultures of Neonatal Mouse Calvariae (Experiment 1) and after Local Injection of Xanthine $(0.25 \mu \mathrm{mol})$ and Xanthine Oxidase $(50 \mathrm{mU})$ over the Scalp of Neonatal Mice In Vivo (Experiment 2). Four Mice Were Injected in Each Treatment Group in Experiment 2.

\begin{tabular}{|c|c|c|c|}
\hline & $\begin{array}{l}\text { Osteoclasts } / \mathrm{mm}^{2} \\
\text { calcified bone } \\
\text { area }\end{array}$ & $\begin{array}{l}\text { Total resorption } \\
\text { surface }\end{array}$ & Base area \\
\hline & & $\%$ & $\mathrm{~mm}^{2} \times 10^{-}$ \\
\hline \multicolumn{4}{|l|}{ Exp. 1 (In vitro) } \\
\hline Control media & $10 \pm 6$ & $7 \pm 3$ & $63 \pm 4$ \\
\hline Control + SOD & $0 \pm 0$ & $0.5 \pm .5^{*}$ & $66 \pm 2$ \\
\hline X/XO & $120 \pm 30^{*}$ & $41 \pm 2^{*}$ & $45 \pm 4^{*}$ \\
\hline $\mathrm{X} / \mathrm{XO}+\mathrm{SOD}$ & $43 \pm 16$ & $15 \pm 2$ & $58 \pm 5$ \\
\hline PTH & $158 \pm 56^{*}$ & $40 \pm 1^{*}$ & $31 \pm 3^{*}$ \\
\hline PTH + SOD & $40 \pm 8$ & $12 \pm 1$ & $61 \pm 4$ \\
\hline IL-1 $\alpha$ & $101 \pm 33^{*}$ & $30 \pm 3^{*}$ & $51 \pm 4$ \\
\hline \multicolumn{4}{|l|}{ Exp. 2 (In vivo) } \\
\hline Saline & $5 \pm 1$ & $0.5 \pm .5$ & $432 \pm 27$ \\
\hline X/XO & $43 \pm 7^{*}$ & $20 \pm 4^{*}$ & $369 \pm 19$ \\
\hline
\end{tabular}

* Significantly $>$ control, $P<0.05$. Statistical differences in Experiment 1 were calculated using Student-Newman-Keuls test, since these data were pooled. Statistical differences in Experiment 2 were calculated using Student's $t$ test.

Bovine PTH 1-34 (Bachem Corp., Torrence, CA) was used at a concentration of $50 \mathrm{ng} / \mathrm{ml}$. Recombinant human IL- $1 \alpha$ (Hoffmann-La Roche, Nutley, NJ) was used at a concentration of $10^{-10} \mathrm{M}$. Superoxide dismutase (SOD) was used at a concentration of $6 \mu \mathrm{M}$.

IL-1 for $48 \mathrm{~h}$, the surfaces were examined using the inverted microscope at low power $(\times 5)$ after staining with NBT. Large NBT-positive cells were seen adjacent to areas of resorption (Fig. $6 \mathrm{~A}$ ). NBT-positive cells were also seen in calvariae incubated with PTH, 1,25 dihydroxyvitamin $\mathrm{D}_{3}$ and TNF (results not shown). In calvariae treated with media alone, NBT-positive cells were so few as to be difficult to detect (Fig. $6 \mathrm{~B}$ ).

We then counted the NBT-positive cells $/ \mathrm{mm}^{2}$ in these calvariae. The number of positively staining multinucleated cells was increased in IL-1-treated calvariae to $38.6 \pm 6.9$ NBT-positive cells $/ \mathrm{mm}^{2}$, compared with $1.6 \pm 0.5 \mathrm{NBT}$-positive cells in calvariae treated in control media. When these resorbing bone cultures were also treated with calcitonin $(5 \mathrm{U} / \mathrm{ml})$, which inhibits osteoclastic bone resorption, substantially reduced numbers of NBT-positive cells were detected (Fig. 7).

When we examined these sections histologically, we confirmed that the large NBT-positive cells that we saw in resorb-

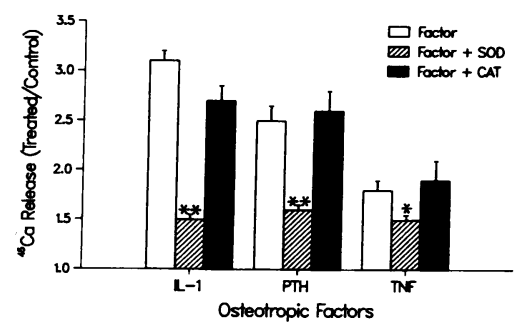

Figure 4. Effects of bovine superoxide dismutase $(6 \mu \mathrm{M})$ and catalase $(6 \mu \mathrm{M})$ on bone resorption in neonatal mouse calvariae stimulated by parathyroid hormone $(50 \mathrm{ng} / \mathrm{ml}), \mathrm{IL}-1 \alpha(0.1$ $\mathrm{nM})$ and tumor necrosis factor $(1 \mathrm{nM})$.

Bone resorbing activity is measured as treated-to-control ratios of ${ }^{45} \mathrm{Ca}$ release. ${ }^{* *}$ Significantly $<$ osteotropic factor alone, $P$ $<0.001$. *Significantly $<$ osteotropic factor alone, $P<0.05$.

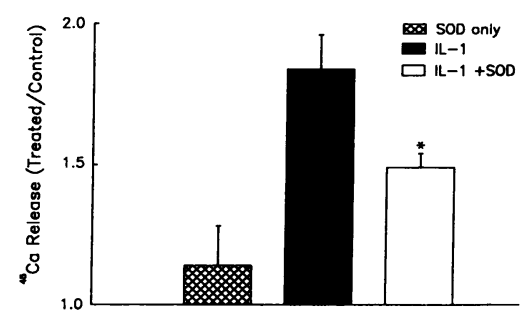

Figure 5. Effects of recombinant human superoxide dismutase $(6$ $\mu \mathrm{M})$ on bone resorption in neonatal mouse calvarial organ cultures stimulated by recombinant human IL- $1 \alpha(0.1$ nM). Bone resorbing activity is measured as treated-to-control ratios of ${ }^{45} \mathrm{Ca}$ release. ${ }^{*}$ Significantly $<\mathrm{IL}-1$ alone, $P<0.05$.

ing calvariae using the inverted microscope were osteoclasts associated with resorption lacunae (Fig. 8). The number of NBT-positive cells lying on the intersect lines in the Zeiss II integrating eyepiece graticule was similar to the number of NBT-positive osteoclasts counted in histological sections of the same calvariae (Fig. 8). Few osteoclasts and no NBT-positive cells were seen in the control bones at this time. Histological sections of the calvariae treated with IL-1 showed that $43 \%$ of the total osteoclasts stained positively with NBT after $48 \mathrm{~h}$. However, there were also a number of NBT-negative multinucleated cells that were associated with resorption lacunae.

Although freshly isolated chicken osteoclasts reduced NBT when adherent to plastic tissue culture dishes, the number and staining intensity of these cells were markedly increased when they were incubated with bone particles for $24 \mathrm{~h}$ (Fig. 9).

When isolated avian osteoclasts were incubated with ferricytochrome $c, 10.4 \pm 2.1 \mathrm{nmol} /$ well per $24 \mathrm{~h}$ of superoxide anion were produced. This could be enhanced by vitamin A (1 $\mu \mathrm{M})(18.0 \pm 1.2 \mathrm{nmol} /$ well per $24 \mathrm{~h})$, and this increase in ferricytochrome $c$ reduction was completely inhibited by superoxide dismutase $(6 \mu \mathrm{M})$.

\section{Discussion}

These data indicate that when free oxygen radicals were generated in the bone environment, osteoclasts were formed, and bone resorption occurred. In addition, they show that the generation of oxygen-derived free radicals was associated with osteoclastic bone resorption stimulated by PTH, IL-1, and TNF. These results were found not only in organ cultures of fetal rat long bones and mouse calvariae, but also in vivo when oxygen radicals were generated adjacent to periosteal bone surfaces. The demonstration that the effects were inhibited by superoxide dismutase, but not by catalase, suggests that the superoxide anion was involved in the resorption of bone that occurred when osteoclasts were stimulated by factors such as PTH, IL-1, and TNF.

NBT is a useful marker that is reduced to a blue insoluble formazan by free radical intermediates. It has been widely used to investigate radical production by activated monocytes and macrophages (13-16). Our results show that osteoclasts have the capacity to reduce NBT, and suggest that osteoclasts are the cells in bone in which radical generation occurs when exposed to bone resorbing hormones or to bone particles. However, even in bone cultures that were resorbing actively, we found that only $43 \%$ of the osteoclasts stained positively for NBT. Why some osteoclasts do not stain with NBT is unclear. One possible explanation is that radical accumulation is a transitory event in the process of osteoclast activation and reflects activity at only one point in time. Thus, the osteoclasts 

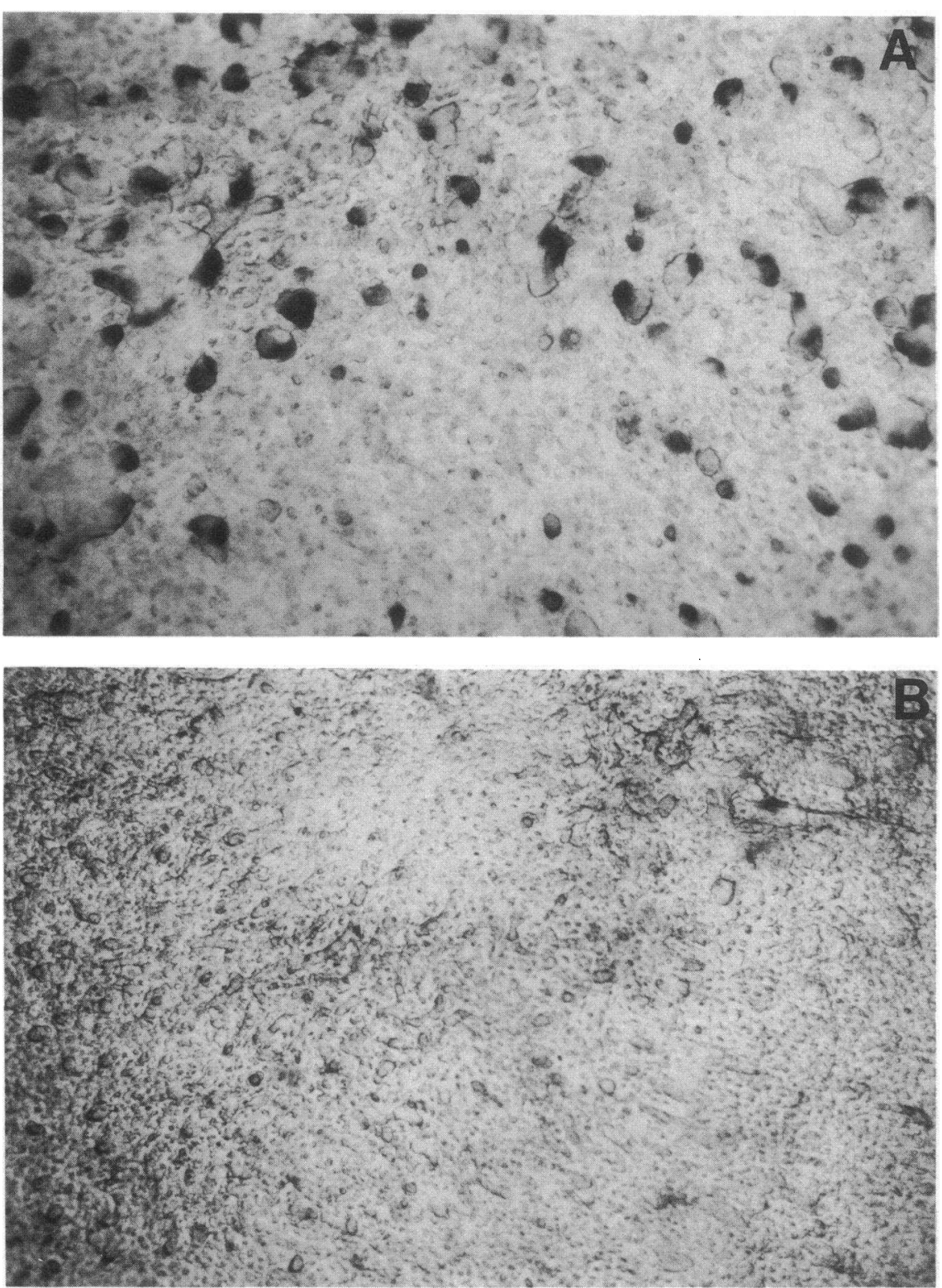

Figure 6. $(A)$ Low power $(5 \times)$ view through inverted microscope of calvarial organ cultures incubated for $48 \mathrm{~h}$ with recombinant human IL- $1 \alpha$ and stained with NBT. Discrete resorption areas are present throughout the surface. Large cells staining positively with NBT were present in the resorption areas. $(B)$ Control cultures for those in $A$. Low power ( $5 \times)$ view through inverted microscope of calvarial organ cultures incubated for $48 \mathrm{~h}$ with control media and stained with NBT. that do not stain positively with NBT may be inactive or have ceased active resorption.

Although the osteoclast has long been recognized as the cell responsible for bone resorption, the mechanisms by which it carries out this function are not fully understood. Recently, it has been suggested that isolated osteoclasts contain superoxide dismutase and produce oxygen radicals in response to hormones (17). Mononuclear phagocytes are capable of consuming and metabolizing oxygen to generate a large array of reactive intermediates capable of inducing cell tissue damage (18). This raises the possibility that the osteoclast may use similar mechanisms in the resorption of bone. The hypothesis that osteoclasts could also cause bone degradation by this process is based on the knowledge that the precursor for the osteoclast probably arises from a colony-forming unit of the granulocyte-macrophage family (CFU-GM), as do cells in the monocyte-macrophage family. Although there have been no reports of the effects of free radicals on the osteoclastic bone resorption process, preliminary evidence from Fallon et al. (17) sug- gests that isolated osteoclasts are capable of producing measurable levels of oxygen-derived free radicals and that these cells contain superoxide dismutase. Our results using NBT staining and ferricytochrome $c$ reduction confirmed those observations. Moreover, work by Stock et al. (19) has shown that chemiluminescence by human monocytes is modulated by calcium-regulating hormones. Since a number of cells produce oxygen radicals in response to certain monokines and lymphokines that also have the capacity of stimulating bone resorption in vitro $(20,21)$, production of free radicals in the bone microenvironment by these factors may be important in the resorption process.

The relationship between active osteoclastic resorption and radical accumulation was confirmed by our studies with calcitonin. We found that calcitonin, which is a selective inhibitor of osteoclast function (22), markedly decreased the number of NBT-positive osteoclasts present in resorbing mouse calvariae. This close relationship between the resorbing osteoclasts and staining with NBT confirms a relationship between oxygen 


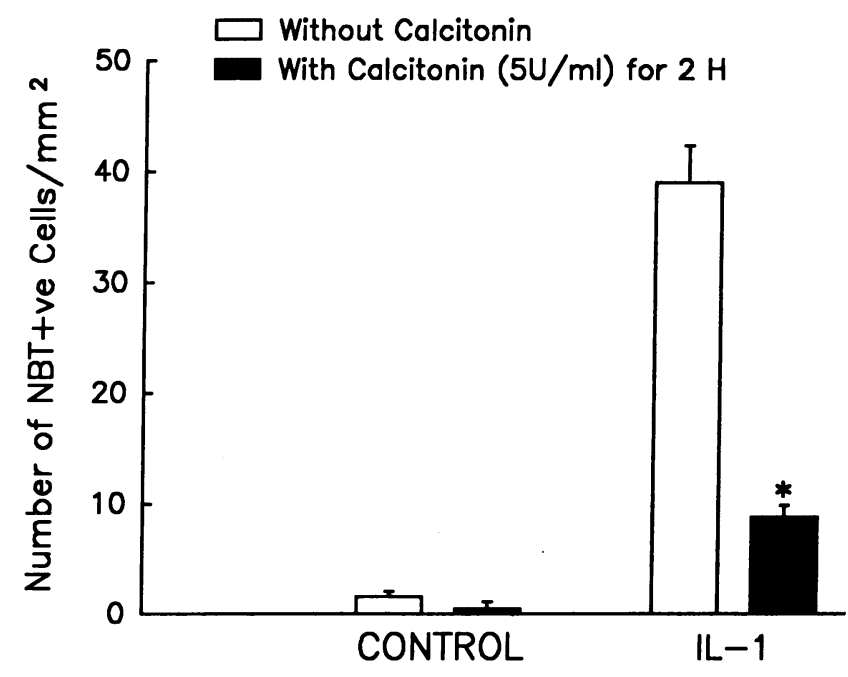

Figure 7. Effects of IL-1 and salmon calcitonin $(5 \mathrm{U} / \mathrm{ml})$ on numbers of NBT-positive cells in neonatal mouse calvariae after $48 \mathrm{~h}$ of organ culture. ${ }^{*}$ Significantly $<$ bones treated with osteotropic factor alone, $P<0.05$.

radicals and active osteoclastic bone resorption. We did not test the effects of calcitonin on isolated mature avian osteoclasts because these cells do not respond reproducibly to calcitonin.

There are several stages in the process of osteoclastic bone resorption at which oxygen-derived free radicals may have an important effect. They could be involved in the degradation of bone matrix under the ruffled border of the osteoclasts. Since free radicals play an important role in many degradative processes, it is attractive to postulate that extracellular tissue components (e.g., bone structural molecules such as collagen and hyaluronic acid) are susceptible to cell-mediated free radical damage. However, our data indicate that the role of oxygenderived free radicals in the process of bone resorption is more complex than this. Since we clearly found an increase in osteoclast numbers and activity when free radicals were generated (Fig. 3, Table I), and that calcitonin inhibited bone re- sorption stimulated by radical generation (Fig. 2), our conclusion is that oxygen-derived free radicals are involved in the complex cellular events involved in formation and activation of the osteoclast itself. This would not be the only situation where reactive oxygen intermediates cause cellular responses. The multiplicity of cellular effects caused by oxygen-derived free radicals have been reviewed extensively (2). Since the molecular events involved in the activation of osteoclasts are complex and poorly understood, but apparently involve several cell types and both primary and secondary signals, the results that we report here have important implications for the resorption of bone that occurs in all physiologic and pathologic states.

It is possible that the generation of oxygen-derived free radicals may be particularly important in the bone resorption that occurs in association with inflammatory diseases. Oxygen-derived species are produced by activated phagocytes including monocytes, macrophages and neutrophils $(23,24)$, and since these cells accumulate adjacent to bone surfaces in chronic inflammatory diseases, radical production by these cells could be responsible for stimulating osteoclast for formation or activation to resorb bone, or even directly enhancing matrix degradation. Chronic inflammatory diseases such as rheumatoid arthritis, periodontal disease and cholesteatoma are associated with collections of chronic inflammatory cells occurring adjacent to bone with subsequent bone destruction. If this turns out to be one of the mechanisms by which bone is destroyed in these circumstances, then drugs that inhibit radical production or scavenge free radicals may be useful therapeutic agents for this type of local bone destruction.

Since the 1950s the generation of free radicals has been implicated in the aging process (25). Age-related changes seen at organ, tissue, and cell levels have focused attention on examining molecular mechanisms that could be responsible. The generation of free radicals has been linked particularly to the age-pigment accumulation and cell loss, which occurs in agerelated diseases such as cancer and atherosclerosis, and to agerelated changes that occur in the brain, the retina, and the skin (26). Since it is well recognized that bone cell function changes with aging, the generation of oxygen-derived free radicals

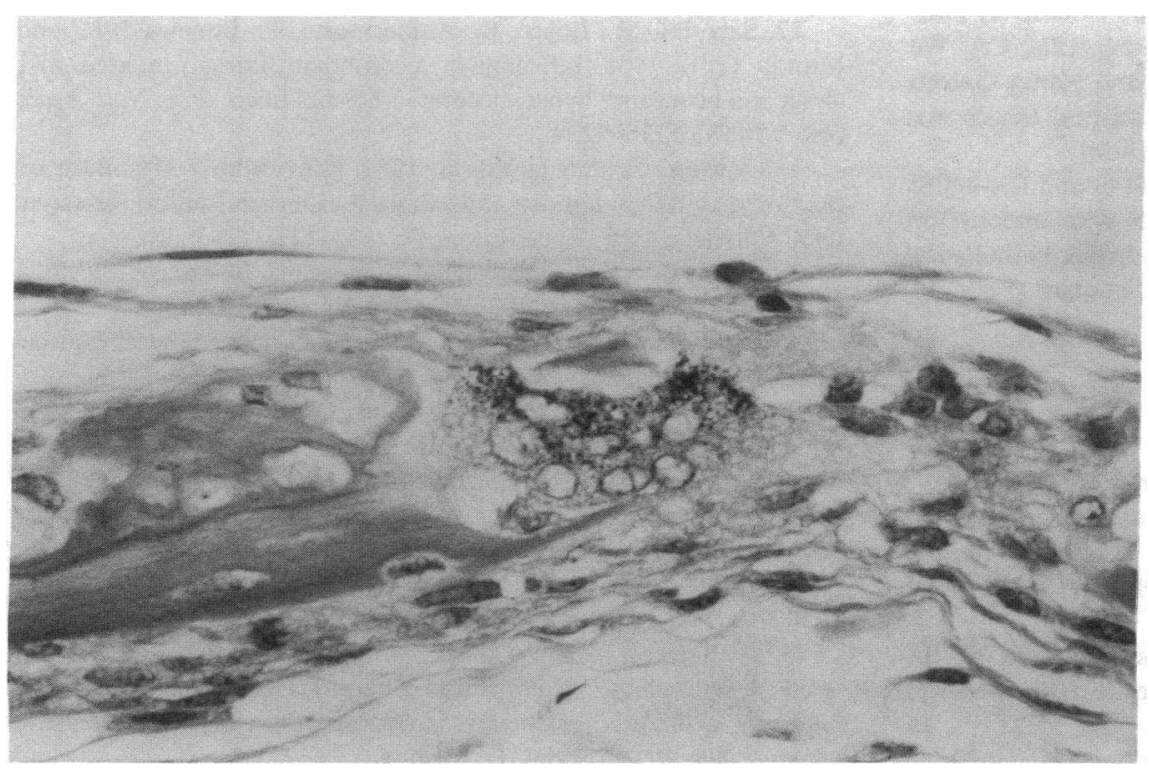

Figure 8. Histologic section through calvarial organ culture after incubation for $48 \mathrm{~h}$ with recombinant human IL- $1 \alpha$ and stained with hematoxylin and eosin and NBT. NBT staining can be seen in an osteoclast in the center of the section. NBT staining was present only in osteoclasts. More than $40 \%$ of the osteoclasts in these bones stained positively with NBT. 


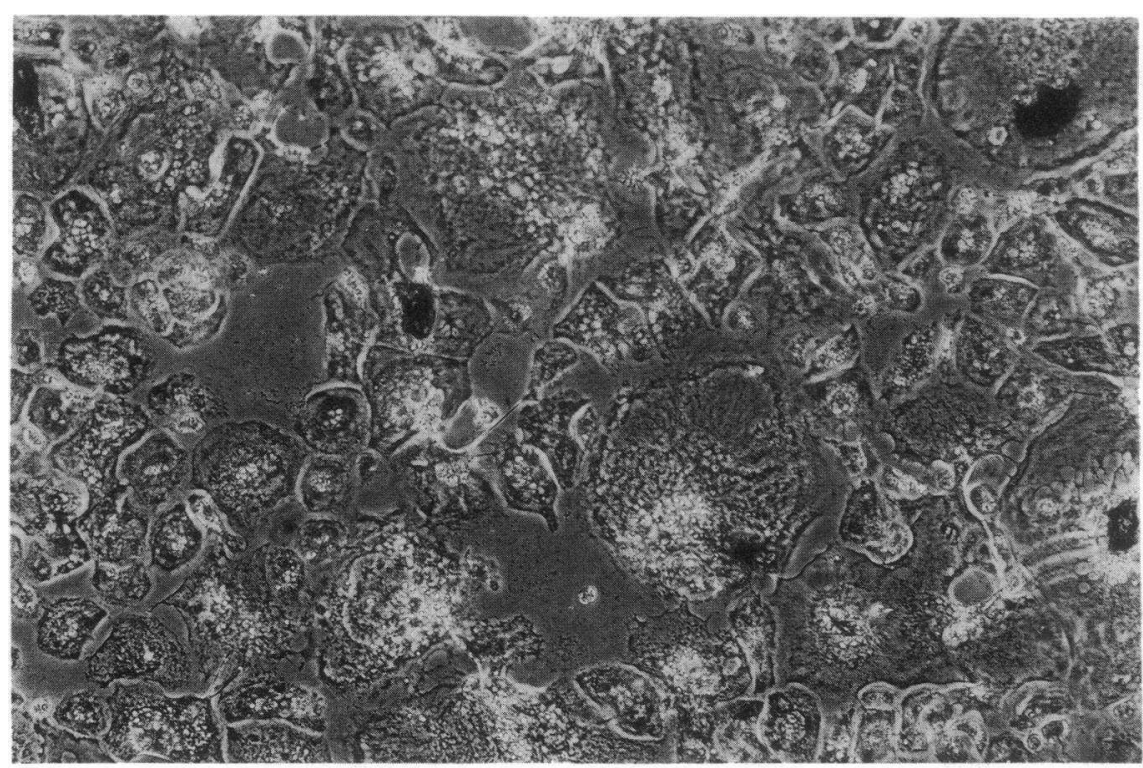

Figure 9. Appearance of isolated avian osteoclasts that have been incubated with bovine bone particles for $24 \mathrm{~h}$ and stained with NBT. Most osteoclasts stained intensely with NBT in presence of bone particles. could conceivably be responsible for some of the changes observed in senescent bone cells.

The mechanisms by which oxygen-derived free radicals are responsible for formation and activation of osteoclasts requires further study. The tools are now available for studying the effects of agents not only on osteoclastic bone resorption but on steps involved in the formation and activation of osteoclasts by using long-term culture techniques (27) or by using isolated osteoclasts (28). It remains to be determined how radicals are responsible for the generation of new osteoclasts, and whether radicals are involved in direct activation of osteoclasts.

\section{Acknowledgments}

Recombinant human interleukin- $1 \alpha$ was kindly provided by Dr. P. Lomedico, Hoffmann-La Roche, Roche Research Center, Nutley, NJ. It was expressed in $E$. coli and extensively purified. Recombinant human tumor necrosis factor, which had been expressed in $E$. coli and extensively purified, was kindly proved by Dr. H. Michael Shepard, Genentech, Inc., South San Francisco, CA. Recombinant human superoxide dismutase was also expressed in $E$. coli and extensively purified. It was kindly supplied by Chiron Corp., Emeryville, CA. We thank Dr. John Chirgwin for helpful discussions and Nancy Garrett and Thelma Barrios for secretarial assistance. Statistical advice was kindly provided by Karen Schulz and Dr. James Dunn.

This research was supported by grants AM-28149 and CA-40035 from the National Institutes of Health. Dr. Boyce was supported in part by the Peel Medical Research Trust, England, The Pathological Society of Great Britain, and the McCunn Trust, Scotland.

\section{References}

1. Mundy, G. R., and G. D. Roodman. 1987. Osteoclast ontogeny and function. In Bone and Mineral Research. V. W. Peck, editor. Elsevier/North Holland Publishing Co., New York. 209-280.

2. Nathan, C. F. 1987. Secretory products of macrophages. J. Clin. Invest. 79:319-326.

3. Halliwell, B., and J. M. C. Gutteridge. 1985. Free Radicals in Biology and Medicine. Clarendon Press, Oxford.

4. Raisz, L. G. 1965. Bone resorption in tissue culture. Factors influencing the response to parathyroid hormone. J. Clin. Invest. 44:103-116.

5. Gowen, M., and G. R. Mundy. 1986. Actions of recombinant interleukin-1, interleukin- 2 and interferon gamma on bone resorption in vitro. J. Immunol. 136:2478-2482.

6. Teti, A., R. O. Oreffo, A. Zambonin-Zallone, and J. T. Triffitt. 1986. Acid phosphatase activity is stimulated in isolated osteoclasts by vitamin A. Boll. Soc. Ital. Biol. Sper. 62:1311-1314.

7. Zambonin-Zallone, A., A. Teti, and M. V. Primavera. 1987. Isolated osteoclast in primary culture: first observations on structure and survival in culture media. Anat. Embryol. 165:405-413.

8. Zambonin-Zallone, A., and A. Teti. 1987. The osteoclast of hen medullary bone under hypocalcaemic conditions. Anat. Embryol. 162:379-392.

9. Fridovich, I. 1970. Quantitative aspects of the production of superoxide anion radicals by milk xanthine oxidase. J. Biol. Chem. 245:4053-4057.

10. McCord, J. M., and I. J. Fridovich. 1968. The reduction of cytochrome c by milk xanthine oxidase. J. Biol. Chem. 243:57535760.

11. Yates, A. J. P., Gutierrez, G. E., Smolens, P., Travis, P. S., Katz, M. S., Aufdemorte, T. B., Boyce, B. F., Hymer, T. K., Poser, J. W., and Mundy, G. R. 1988. Effects of a synthetic peptide of a parathyroid hormone-related protein on calcium homeostasis, renal tubular calcium reabosorption and bone metabolism. J. Clin. Invest. 81:932-938.

12. Sabatini, M., Boyce, B., Aufdermorte, T., Bonewald, L., and Mundy, G. R. 1988. Infusions of recombinant human interleukin-1 alpha and beta cause hypercalcemia in normal mice. Proc. Natl. Acad. Sci. USA. 85:5235-5239.

13. Auclair, C., and E. Voisin. 1983. Nitro Blue Tetrazolium reduction. In CRC Handbook of Methods for Oxygen Radical Research. CRC Publishing Co., Boca Raton, FL. 123-132.

14. Rook, G. A. W., J. Steele, S. Umar, and H. M. Dockell. 1985. A simple method for the solubilization of reduced NBT and its use as a colormetric assay for activation of human macrophages by gamma-interferon. Immunol. Methods. 82:161-167.

15. Murray, H. W., and Z. A. Cohn. 1980. Macrophage oxygendependent antimicrobial activity. III. Enhanced oxidative metabolism as an expression of macrophage activation. J. Exp. Med. 152:15961609.

16. Murray, H. W., and Z. A. Cohn. 1980. Mononuclear phagocyte antimicrobial and antitumor activity: The role of oxygen intermediates. J. Invest. Dermatol. 74:285-287.

17. Fallon, M., S. Silverton, P. Smith, T. Moskal, C. Constantinescu, R. Feldman, E. Golub, and I. Shapiro. 1986. The oxidative metabolism of isolated osteoclasts is regulated by calcitropic agents. $J$. Bone Miner. Res. 1(Suppl. 1). Abstr. 1. 
18. Babior, B. M. 1984. Oxidants from phagocytes: agents of defense and destruction. Blood. 64:959-966.

19. Stock, J. L., J. A. Coderre, and P. H. Levine. 1984. Effects of calcium-regulating hormones and drugs on monocyte chemiluminescence. J. Clin. Endocrinol. Metab. 55:956-960.

20. Freund, M., and E. Pick. 1986. The mechanism of action of lymphokines IX. The enzymatic basis of hydrogen production by lymphokine-activated macrophages. J. Immunol. 137:1312-1318.

21. Clement, L. T., and J. E. Lehmener. 1983. Regulation of the growth and differentiation of human monocytic cell lines by lymphokines. Introduction of synovial avion production and chemiluminescence. J. Immunol. 130:2763-2766.

22. Friedman, J., W. Y. W. Au, and L. G. Raisz. 1968. Responses of fetal rat bone to thyrocalcitonin in tissue culture. Endocrinology. 82:149-156.

23. Weiss, S. J., and A. F. LoBoglio. 1982. Biology of disease phagocyte-generated oxygen metabolites and cellular injury. Lab. Invest. 47:5-17.
24. Babior, B. M. 1984. The respiratory burst of phagocytes. J. Clin. Invest. 73:599-601.

25. Harman, D. 1983. Free radicals and the origination, evolution, and present status of the free radical theory of aging. In Free Radicals in Molecular Biology, Aging, and Diseases. D. Armstrong, R. S. Sohal, R. G. Cutler, and T. F. Slater, editors. Raven Press, New York. 1-12.

26. Armstrong, D. 1983. Free radical involvement in the formation of lipopigments. In Free Radicals in Molecular Biology, Aging, and Disease. D. Armstrong, R. S. Sohal, R. G. Cutler, and T. F. Slater, editors. Raven Press, New York. 129-142.

27. Takahashi, N., B. R. MacDonald, J. Hon, M. E. Winkler, R. Derynck, G. R. Mundy, and G. D. Roodman. 1986. Recombinant human transforming growth factor alpha stimulates the formation of osteoclast-like cells in long term human marrow cultures. J. Clin. Invest. 78:894-898.

28. Thomson, B. M., G. R. Mundy, and T. J. Chambers. 1987. Tumor necrosis factors $\alpha$ and $\beta$ induce osteoblastic cells to stimulate osteoclastic bone resorption. J. Immunol. 138:775-779. 